\section{EBM 2005plus}

Helga Peter ${ }^{1}$ und Thomas Penzel ${ }^{2}$

${ }^{1}$ Marburg, Deutschland

${ }^{2}$ Interdisziplinäres Schlafmedizinisches Zentrum, Charité Universitätsmedizin Berlin, Berlin, Deutschland

\section{Definition}

Aktueller einheitlicher Bewertungsmaßstab zur Festlegung der Höhe der Vergütung für Vertragsärzte und -psychotherapeuten aus dem Jahr 2005.

Siehe $\triangleright$,Indikationsbezogenes ambulantes Monitoring“. 